\title{
Analisando a Participação e a Aprendizagem em Comunidades Virtuais a partir da Teoria de Barbara Rogoff.
}

\author{
Raphael A. Dourado - CIn-UFPE, rasd2@ cin.ufpe.br \\ Candy E. Marques-Laurendon - PPG Psicologia Cognitiva-UFPE, nacyda@gmail.com \\ Rodrigo Lins Rodrigues - Dept. Educação-UFRPE, rodrigo.linsrodrigues@ufrpe.br \\ Alex Sandro Gomes - CIn-UPE, asg@ cin.ufpe.br
}

\begin{abstract}
Resumo. O avanço nas telecomunicações tem viabilizado formas cada vez mais sofisticadas de interação social entre indivíduos geograficamente distantes. Estes recursos têm sido utilizados para diversos fins, dentre eles viabilizar o surgimento das comunidades virtuais de aprendizagem. No entanto, ainda há uma carência de instrumentos que auxiliem na avaliação processual da aprendizagem nestes contextos. Neste artigo, é discutida a adequabilidade da teoria sociocultural de Barbara Rogoff, em especial a noção dos "planos de análise", como estratégia para avaliar de forma processual a aprendizagem em comunidades virtuais. São apresentados os principais construtos da teoria, os trabalhos encontrados na literatura que a utilizam no contexto de comunidades virtuais, e as possibilidades de investigação ainda não exploradas.
\end{abstract}

Palavras-chave: avaliação processual; comunidades virtuais; Barbara Rogoff.

\begin{abstract}
The developments in telecommunications has enabled increasingly sophisticated forms of social interaction between geographically distant individuals. These resources have been used for various purposes, including supporting the emergence of virtual learning communities. However, there is a need for instruments to assist with process-oriented assessments in these contexts. In this article, we discuss the suitability of Barbara Rogoff's sociocultural theory, especially the notion of the "plans of analysis", as a strategy for process-oriented assessment of learning in virtual communities. We present the theory's main constructs, the studies that use them in the context of virtual communities, and the possibilities of research still unexplored.
\end{abstract}

Keywords: process-oriented assessment; virtual communities; Barbara Rogoff.

\section{INTRODUÇÃO}

Interações sociais distais no tempo ou no espaço são possíveis, pelo menos, desde a invenção da escrita, por meio da qual um sujeito pode interagir com outro de forma assíncrona (Rogoff, 1998b:726). No entanto, os avanços nas tecnologias de telecomunicação como o rádio, o telégrafo, a televisão e a internet têm tornado estes tipos de interação cada vez mais ricas e próximas da experiência presencial, especialmente pela possibilidade de comunicação síncrona entre pares. Desse modo, 
muitos autores têm proposto aplicar o conceito tradicional de comunidade para os

grupos de indivíduos que interagem por meio destas tecnologias, dando origem ao conceito de comunidades virtuais (Porter, 2004).

De acordo com Porter (2004), uma comunidade virtual pode ser definida como um grupo de indivíduos e/ou parceiros de negócios que interagem em torno de interesses em comum, sendo que esta interação é ao menos parcialmente suportada ou mediada por tecnologias, e guiada por determinados protocolos ou normas. Nesta definição, é importante notar que as interações em uma comunidade virtual não precisam ser exclusivamente virtuais, podendo incluir também interações presenciais. Desse modo, as comunidades virtuais possuem diferentes níveis de "virtualidade" (Virnoche \& Marx, 1997).

Henri e Pudelko (2003) propõem uma classificação das comunidades virtuais em quatro tipos (ou estágios), distribuídos ao longo de um contínuo, de acordo com o grau de intencionalidade e a força da ligação social de seus membros. Um dos estágios definido pelos autores é o de "comunidade de aprendizes", caracterizado por indivíduos envolvidos em atividades com fins pedagógicos, propostas por sujeitos mais experientes (instrutores/tutores), e atreladas a um currículo. O tempo de vida destas comunidades é restrito à duração do programa educacional, e como o objetivo declarado (interesse em comum) das mesmas é o aprendizado, a avaliação da aprendizagem assume grande importância.

No entanto, são inúmeros os desafios para avaliar adequadamente a aprendizagem de sujeitos participando numa comunidade virtual (Koç et al, 2015). Dentre eles, há a escassez de instrumentos que auxiliem na análise do processo de aprendizagem nestes ambientes, o que contribui para restringir as avaliações aos produtos da aprendizagem (Bonanno, 2015). Para superar este desafio, o primeiro passo é adotar uma perspectiva teórica que entenda a aprendizagem como um processo contínuo, e a partir desta perspectiva propor novos instrumentos de apoio à avaliação processual.

Nesse sentido, a teoria do desenvolvimento de Barbara Rogoff (1990; 1998a; 2005) surge como uma alternativa promissora. Fortemente influenciada pelos trabalhos de Lev Vygotsky (Mello, 2004), Rogoff desenvolve a ideia de que a aprendizagem e o desenvolvimento ocorrem por meio da participação variável em atividades culturais, e propõe analisar esta participação através de três planos distintos, porém mutuamente constitutivos: os planos institucional, interpessoal e pessoal (Rogoff, 1990; Rogoff, 1998a). Mas embora os planos de análise propostos por Rogoff prevejam a possiblidade de interações não-presenciais (Rogoff, 1998b:726), ainda são escassos os estudos que os utilizem neste contexto. Assim, este artigo se propõe a discutir a adequabilidade da teoria de Barbara Rogoff como alicerce conceitual para a construção de novos mecanismos de apoio à avaliação processual em comunidades virtuais de aprendizagem.

O restante deste texto está organizado da seguinte maneira: a Seção 2 apresenta em detalhes os planos de análise propostos por Barbara Rogoff; a Seção 3 descreve os trabalhos encontrados na literatura que utilizam a teoria da autora no contexto das comunidades virtuais; a Seção 4 discute as lacunas de pesquisa identificadas e propõe possibilidades de investigações futuras; e por fim a Seção 5 traça as considerações finais do trabalho. 


\section{A TEORIA SOCIAL DE BARBARA ROGOFF}

Um pressuposto central na teoria de Barbara Rogoff é a visão do desenvolvimento humano como resultado da participação contínua em atividades culturais. Essa participação contribuiria tanto para a transformação do indivíduo quanto das práticas de sua comunidade ao passar das gerações (Rogoff, 2005:41). Assim, percebe-se o alinhamento de sua teoria com a visão sociocultural de que os processos individuais e culturais são mutuamente constitutivos.

No entanto, a autora propõe uma nova abordagem para analisar a atividade sociocultural. Rogoff critica abordagens hierárquicas ou segmentadas, e também aquelas que ignoram a interdependência dos elementos que a constituem. Utilizando o conceito de "atividade" ou "acontecimento" (Leontiev, 1981) como unidade básica de análise, a autora propõe um modelo para analisar a atividade cultural (e o desenvolvimento) baseado em três planos distintos, porém inseparáveis: cultural/institucional, interpessoal e pessoal (Rogoff, 1998a). Rogoff argumenta que é possível descrever as estruturas que constituem uma atividade ou acontecimento sem "perder a marca da interdependência inerente ao conjunto" (Rogoff, 1998a:124), da mesma forma que é possível descrever o funcionamento de um determinado órgão do corpo humano sem ignorar suas relações com os demais órgãos.

A cada um destes planos, a autora associa processos desenvolvimentais (também interdependentes e inseparáveis), a saber: aprendizado (plano cultural/institucional), participação guiada (plano interpessoal) e apropriação participatória (plano pessoal). O restante desta seção apresenta os três planos de análise e seus conceitos associados.

\subsection{O plano cultural/institucional}

O plano cultural/institucional (ou comunitário) enfoca a "estrutura institucional e as tecnologias culturais da atividade intelectual” (Rogoff, 1998a:126). Neste plano de análise, Rogoff se apoia na "metáfora da criança enquanto aprendiz" (Santana et. al., 2006) para definir o conceito de aprendizado como um processo que envolve indivíduos ativos, participando em uma atividade culturalmente organizada, que tem como parte de seu propósito a evolução da participação responsável por pessoas menos experientes (Rogoff, 1998a). Pode-se perceber que este conceito amplia a ideia de aprendizado para qualquer atividade culturalmente organizada, e não apenas atividades de instrução explícita em contexto escolar. Até mesmo o aprendizado de habilidades "indesejáveis" - como a violência - está abarcado por este conceito.

Além disso, o conceito de aprendizado proposto por Rogoff vai além da dualidade experiente-inexperiente. $\mathrm{Na}$ sua visão, todos aprendem, inclusive os mais experientes, que "ainda estão desenvolvendo habilidade e entendimento no processo de engajar-se em atividades com outros de experiências variadas" (Rogoff, 1998a:126). Neste sentido, o aprendizado envolve o engajamento em atividades culturalmente organizadas, onde os aprendizes "se tornam participantes mais responsáveis" (ibid).

\subsection{O plano interpessoal}

O plano interpessoal é constituído pelos acontecimentos cotidianos, incluindo: interação direta com outros, dedicar-se (ou evitar) atividades designadas, possibilitadas, ou impossibilitadas por outros, mesmo que estes "outros" não estejam fisicamente 
presentes, ou até mesmo não saibam da existência um do outro - como o autor de um livro e seus leitores, mostrando assim a possibilidade de utilizar a teoria de Rogoff para analisar interações não-presenciais (distais), como detalhado em Rogoff (1998b:726). Para este plano, Rogoff propõe o conceito de participação guiada, entendido como os processos e sistemas de envolvimento entre as pessoas à medida que elas se comunicam e coordenam esforços ao participar de atividades de cunho cultural, ou seja, "o envolvimento mútuo dos indivíduos e seus companheiros sociais” (Rogoff, 1998a:129). Aqui, "participação" é entendido como a observação e envolvimento prático em uma atividade, enquanto "guiada" enfatiza a direção oferecida por valores culturais e sociais, bem como por parceiros sociais.

No entanto, o conceito de participação guiada não pressupõe necessariamente uma ação simétrica, nem sequer em conjunto. Por exemplo, ao trabalhar sozinha num relatório, um estudante está participando de uma atividade cultural com orientação, que envolve "interações com o professor, colegas, membros da família, bibliotecários e autores, a indústria de editoramento (...)" (Rogoff, 1998a:130). Todos estes agentes, mesmo de forma não-simultânea, auxiliam na direção/orientação da atividade. Dessa forma a participação pode ser tanto tácita quanto implícita, face-a-face ou longínqua, e envolvendo pessoas conhecidas ou desconhecidas.

A ideia de participação defendida por Rogoff está alinhada com a perspectiva da mediação de Vygotsky, porém considera aspectos que não eram tão centrais na teoria deste último. Dentre estes aspectos, pode-se citar as interações de crianças em comunidades onde a cultura escolar ocidental não é fortemente valorizada, e as interações de crianças de classe média em atividades cognitivas do dia-a-dia em casa e com os vizinhos.

Por fim, é importante mencionar que um conceito similar ao de participação guiada foi proposto por Lave e Wenger (1991). Trata-se da ideia de "participação periférica legítima", segundo a qual a aprendizagem ocorre com base no envolvimento variável das pessoas numa comunidade, que se inicia no que o autor chama de "periferia". Rogoff (2005:233) menciona esta similaridade, ao explicitar este conceito de Lave e Wenger.

\subsection{O plano pessoal}

O plano de análise pessoal coloca o indivíduo "na condição de foco de análise" (Rogoff, 2005:55), mas sem ignorar os aspectos interpessoais e culturais envolvidos na atividade. Neste plano, Rogoff introduz o conceito de apropriação participatória, central em sua teoria, que se refere a "como os indivíduos mudam através do seu envolvimento em uma ou outra atividade" (Rogoff, 1998a:126). Ou seja, por meio da participação em atividades culturais, os indivíduos se modificam e tornam-se preparados para desenvolver outas atividades similares no futuro.

No entanto, o conceito de apropriação não deve ser confundido com "internalização", ou seja, como transposição de algo estático de um campo externo para o interno. Enquanto a ideia de internalização traz suposição de que há "entidades estáticas envolvidas na 'aquisição' de conceitos, memórias, conhecimentos, habilidades, etc.” (Rogoff, 1998a:133), a abordagem da apropriação participatória não enxerga a cognição como um grupo de pertences armazenados, mas sim “como processos ativos 
que não podem ser reduzidos à possessão de objetos" (ibid), divergindo assim da perspectiva vygotyskyana clássica.

Ainda assim, Rogoff afirma que muitas vezes o termo "apropriação" é utilizado na literatura com sentidos distintos do que ela atribui à apropriação participatória. Rogoff apresenta três usos distintos do termo apropriação (Rogoff, 1998a:134). O primeiro seria como sinônimo de "internalização", ou seja, algo externo é "importado" para a estrutura cognitiva do indivíduo. O segundo uso vai um pouco além da ideia de internalização, mas Rogoff ainda o considera como uma variação deste: consiste na visão de que algo externo é importado e transformado durante o processo, de forma que se adeque aos propósitos do novo possuidor.

O terceiro uso do termo "apropriação" refere-se à noção de apropriação participatória apresentada por Rogoff. Aqui, o próprio limite entre externo e interno é questionado, já que "uma pessoa que esteja participando em uma atividade é parte desta atividade e não algo separado dela" (Rogoff, 1998a:134). Ou seja, o conceito de apropriação participatória rejeita a ideia de que o mundo social é externo ao indivíduo. Nesse sentido, "a apropriação ocorre no processo de participação à medida que o indivíduo se modifica através do envolvimento na situação em questão" (ibid). Essa participação contribuiu tanto para a direção da situação atual quanto para preparar o indivíduo para envolver-se em situações futuras, explicando a possibilidade de transferência de aprendizagem.

\subsection{Exemplo de utilização dos três planos para analisar uma atividade sociocultural}

Para exemplificar como os três planos podem ser utilizados para analisar uma atividade sociocultural, será apresentado um estudo de caso descrito em Rogoff (1998a), onde a autora analisa a atividade anual de venda de doces pelas Escoteiras da América. Do ponto de vista do plano institucional/cultural, trata-se de um evento anual para levantamento de fundos para a instituição — uma organização voluntária dedicada à educação de meninas. Neste evento, as meninas, ora apoiadas por adultos ora por suas colegas, planejam as atividades, registram os pedidos, e realizam as entregas dos doces.

Já analisando a partir do plano interpessoal, a autora observa o processo de participação guiada através das várias combinações (arrangements) entre os envolvidos na atividade. Os doces são vendidos e entregues com a ajuda de um parceiro, normalmente outra escoteira, um parente próximo ou um dos pais. Parceiros mais jovens atuam mais frequentemente na fase de venda, enquanto parceiros adultos são mais comuns na fase de entrega, que envolvia coleta de dinheiro e entrega de grandes volumes de mercadorias. No geral, observou-se que o balanço de responsabilidades oscilava no decorrer do período de entrega. Além disso, observou-se também que a participação guiada incluiu tanto combinações e interações com fins explícitos de instruir - como treinamentos recebidos pelas garotas - como atividades que não tinham qualquer intenção de instrução ou auxílio - conversas com clientes, argumentação com companheiras para coordenar as atividades, etc.

Por fim, do ponto de vista do plano pessoal, é possível observar o processo de apropriação participatória a partir das mudanças em "como as meninas participavam em diferentes aspectos da atividade" (Rogoff, 1998a:139). Segundo a autora, estas mudanças puderam ser observadas em vários aspectos: no cálculo dos preços para os clientes, responsabilidade que de início era primordialmente dos pais, mas ao decorrer 
do tempo foi sendo assumida pelas meninas; no planejamento da entrega das encomendas, ao passo que as meninas foram se tornando mais íntimas com o planejamento das rotas, otimização de recursos e coordenação dos adultos que as auxiliavam; e no preenchimento dos formulários de pedidos (fornecidos pela organização).

Após esta breve apresentação da teoria de desenvolvimento de Barbara Rogoff, serão apresentados a seguir alguns trabalhos existentes na literatura que se apoiam nesta perspectiva teórica para analisar a participação em comunidades virtuais de aprendizagem.

\section{ANALISANDO COMUNIDADES VIRTUAIS DE APRENDIZAGEM À LUZ DA TEORIA DE BARBARA ROGOFF}

Como discutido na Subseção 2.2, o conceito de participação guiada proposto por Rogoff não está restrito a interações presenciais e simultâneas. Desse modo, existe espaço em sua teoria para acomodar a análise da participação em comunidades virtuais de aprendizagem, onde a interação é total ou parcialmente mediada por tecnologias. Nesta seção, são discutidos alguns trabalhos encontrados na literatura que utilizaram a teoria de Rogoff para analisar a aprendizagem nestes contextos.

No trabalho desenvolvido por Heo (2008), o autor utiliza os planos de análise de Rogoff em conjunto com a noção de Comunidades de Prática (Lave \& Wenger, 1991; Wenger, 1998) para investigar como a aprendizagem ocorre em uma comunidade informal de aprendizes voltada a profissionais da indústria alimentícia, onde a participação é voluntária e espontânea. A comunidade analisada possuía mais de 40.000 usuários registrados, e oferecia serviços como fórum de discussão, oportunidades de emprego, boletins de notícia e relatórios de mercado.

O autor deste trabalho estrutura sua análise dos três planos propostos por Rogoff da seguinte forma: no plano comunitário, procura identificar como o aprendizado ocorre na comunidade virtual estudada; no plano interpessoal, como o aprendizado ocorre entre os participantes desta comunidade; e no plano pessoal, como cada participante aprende através da participação nesta comunidade. Do ponto de vista metodológico, o autor utiliza vários métodos provenientes da análise etnográfica virtual - uma variante da análise etnográfica tradicional, aplicada a ambientes virtuais (Hine, 2000). Para analisar o plano comunitário, foi utilizado o framework descritivo de Comunidades e Prática proposto por Wenger (1998); para analisar o plano interpessoal, foram utilizadas técnicas de análise do discurso mediado por computador (Computer-mediated Discourse Analysis - CMDA; Herring, 2004), pelo fato de o processo de participação ser mediado pelo discurso; já para o plano pessoal de análise, foram utilizados questionários, entrevistas virtuais e observação etnográfica a respeito da percepção dos usuários sobre seu aprendizado, e as transformações de papéis durante sua participação na comunidade.

Por meio deste estudo, o autor conseguiu identificar padrões de interação interessantes na comunidade virtual (Heo \& Breuleux, 2009). Um deles foi o "padrão cíclico", que demonstra a importância do indivíduo que iniciou a interação — no contexto deste trabalho, um tópico num fórum virtual - estar ativamente engajado no acompanhamento e mediação da discussão para que sua dúvida ou problema seja resolvido. 
Outro estudo que fez amplo uso da teoria de Barbara Rogoff para analisar comunidades virtuais de aprendizes foi desenvolvido por Gray \& Tatar (2004). Neste trabalho, os autores analisaram a trajetória de um indivíduo, Robert, em seu envolvimento com uma comunidade virtual de aprendizagem (TappedIn), e as mudanças que este envolvimento trouxe para sua prática profissional. Segundo os autores, a decisão de utilizar os planos de análise propostos por Rogoff neste estudo se deu pela sua capacidade de acomodar construtos de diversas outras teorias correlatas, como a Teoria da Atividade (Leontiev, 1981) e Comunidades de Prática (Wenger, 1998).

No entanto, os autores deste estudo propõem acrescentar um quarto plano de análise à teoria, a tecnologia, como forma de acomodar questões peculiares dos ambientes virtuais. Desse modo, o plano tecnologia é definido como o uso das tecnologias da informação e comunicação (TICs) por indivíduos como forma de construir significado. Segundo o autor, da mesma forma que os outros planos, a tecnologia não deve ser entendida como existindo separadamente, mas sim como um aspecto profundamente inter-relacionado com os planos comunitário/cultural, interpessoal e pessoal. Porém, numa perspectiva sócio-histórico-cultural, a tecnologiana acepção abrangente do termo, incluindo não apenas a tecnologia da informação, mas todos os demais tipos de tecnologia - é parte da cultura, pois é produto da mesma. Em Rogoff (2005:225), por exemplo, a autora analisa a tecnologia da escrita em papel como inserida no plano cultural/institucional, pois é produto de um longo processo cultural de aperfeiçoamento, que remonta a invenção do papiro no antigo Egito. Desse modo, não parece necessário definir um novo plano de análise para acomodar a tecnologia.

Partindo dos quatro planos de análise adotados, e utilizando como metodologia o estudo de caso etnográfico, os autores buscaram identificar as interconexões entre os contextos online e offline na trajetória profissional de Robert, e se o contexto sociocultural da aprendizagem online é mais flexível e adaptativo que o off-line, ou apenas o reproduz. No plano pessoal, os autores analisaram como a trajetória de vida de Robert - que havia se mudado dos EUA para a França - está entrelaçada com suas interações na comunidade virtual TappedIn, por meio da qual ele transforma sua participação tanto na comunidade virtual quanto na sua comunidade local. No plano interpessoal os autores identificaram como os recursos do sistema TappedIn possibilitam diferentes tipos de interações interpessoais (conversas privadas entre dois sujeitos, conversas em grupo, notificações, etc.). Já no plano comunitário, os autores analisam como o desenvolvimento de Robert estende-se para além de si mesmo e de suas relações interpessoais, provocando mudanças tanto na estrutura das atividades da comunidade virtual TappedIn quanto em instituições da região em que vive. Por fim, no plano tecnológico, os autores investigam como a infraestrutura do sistema que suporta a comunidade TappedIn possibilita e restringe o desenvolvimento dos demais planos, e como a própria tecnologia se desenvolve paralelamente aos indivíduos que a utilizam.

Embora outros estudos na literatura relacionem a teoria de Barbara Rogoff ao contexto das comunidades virtuais (Kleinsasser et. al., 2016; Borge \& Goggins, 2014; Khoo et. al., 2009; Montiel-Overall, 2010), apenas os três trabalhos discutidos nesta seção (Heo, 2008; Heo \& Breuleux, 2009; Gray \& Tatar, 2004) utilizaram efetivamente a noção dos planos de análise como fundamento metodológico de suas pesquisas. 


\section{DISCUSSÃO}

Os trabalhos apresentados na Seção 3 apontam a viabilidade de se utilizar os construtos propostos por Barbara Rogoff no contexto de comunidades virtuais. A partir de alguns ajustes metodológicos - como a utilização de técnicas de análise do discurso mediado por computador e etnografia digital —, os autores conseguiram analisar a participação em plataformas digitais através dos três planos propostos por Rogoff, e identificar os processos desenvolvimentais associados: aprendizagem, participação guiada e apropriação participatória.

No entanto, não foram encontrados trabalhos que utilizassem efetivamente métodos quantitativos para analisar a participação em comunidades virtuais sob a ótica da teoria de Rogoff. Especialmente em ambientes virtuais de aprendizagem (AVAs), onde há a possibilidade de capturar e armazenar automaticamente grandes quantidades de dados relativos à interação dos sujeitos com o ambiente, abordagens quantitativas como o Learning Analytics podem auxiliar na rápida identificação de padrões de comportamento na comunidade, bem como explicitar a natureza processual da aprendizagem nestes ambientes, indo além da análise dos produtos da interação (Siemens \& Gasévic, 2012; Bogarín et al, 2018).

Dentre as diversas abordagens quantitativas que podem ser usadas neste contexto, as interfaces interativas de visualização de dados têm se mostrado uma opção promissora (Vieira et al, 2018). Este tipo de artefato se apoia na elevada capacidade de percepção visual humana para comunicar de forma rápida e intuitiva conjuntos de dados extensos e complexos. Além disso, os dados a serem visualizados podem ser préprocessados através de técnicas estatísticas e pelo uso de inteligência computacional, de forma a oferecer análises mais sofisticadas como predições e inferências (Peña-Ayala, 2014).

\section{CONSIDERAÇÕES FINAIS}

Neste artigo, foi apresentada uma breve revisão da teoria de Barbara Rogoff, abordando um de seus principais conceitos: a análise da atividade sociocultural por meio de três planos distintos, porém interdependentes: comunitário/institucional, interpessoal e pessoal. Além disso, foram discutidos trabalhos encontrados na literatura que se propuseram a aplicar a teoria de Rogoff para a análise de comunidades virtuais de aprendizagem, contexto este que não é explorado pela autora em seus trabalhos. Por fim, este estudo identificou a ausência de trabalhos que utilizem métodos quantitativos para analisar a atividade sociocultural através da perspectiva proposta por Rogoff, o que constitui uma lacuna a ser explorada em pesquisas futuras.

\section{REFERÊNCIAS BIBLIOGRÁFICAS}

BOGARÍN, A.; CEREZO, R.; ROMERO, C. A survey on educational process mining. Wiley Interdisciplinary Reviews: Data Mining and Knowledge Discovery, v. 8, n. 1, 2018.

BONANNO, P. Assessing Technologyenhanced Learning: A Process-Oriented Approach, 2015. In: KOÇ, Selma; LIU, Xiongyi; WACHIRA, Patrick (Ed.). Assessment in online and blended learning environments. IAP, 2015. 
BORGE, M.; GOGGINS, S. Towards the facilitation of an online community of learners: Assessing the quality of interactions in Yammer. Proc of ICLS, 14, 753$761,2014$.

GRAY, J. H.; TATAR, D. Sociocultural analysis of online professional development: A case study of personal, interpersonal, community, and technical aspects. In "Designing for Virtual Communities in the Service of Learning". New York: Cambridge University Press, 2004.

HEO, G. M.; BREULEUX, A. Roles of initiators and interaction patterns: Exploring an informal online community at the interpersonal plane. Proceedings of the Computer Supported Collaborative Learning 2009 Conference, 13-17, 2009.

HEO, G. M. Learning in an Informal Web-Based Community of Practice: A Study of Community, Interpersonal, and Individual Planes. PhD Thesis: McGill University, Montreal, Quebec, Canada, 2008.

HENRI, F.; PUDELKO, B. Understanding and analysing activity and learning in virtual communities. Journal of Computer Assisted Learning, v. 19, n. 4, p. 474487, 2003.

HERRING, S. C. Computer-mediated discourse analysis: An approach to researching online behavior. In Barab, S. A.; King, R. \& Gray, J. H. (Eds.), Designing for virtual communities in the service of learning. United Kingdom: Cambridge University Press, 2004.

HINE, C. Virtual ethnography. Sage Publications Ltd, 2000.

KHOO, E.; FORRET, M.; COWIE, B. Developing an online learning community: A model for enhancing lecturer and student learning experiences. ASCILITE 2009 The Australasian Society for Computers in Learning in Tertiary Education, 528-532, 2009.

KLEINSASSER, R.; HONG, Y. C. Online Group Work Design: Processes, Complexities, and Intricacies. TechTrends, 60(6), 569-576., 2016.

KOÇ, S.; LIU, X.; WACHIRA, P. (Ed.). Assessment in online and blended learning environments. IAP, 2015.

LAVE, J.; WENGER, E. Situated Learning: Legitimate Periperal Participation. Cambridge, UK: Cambridge University Press, 1991.

LEONTIEV, A. N. The problem of activity in psychology. In J. V. Wertsch (Ed.), The concept of activity in Soviet psychology. Armonk, NY: Sharpe, 1981.

MELlO, S. A. A Escola de Vygotsky. In: CARRARA, K. (Org.) Introdução à psicologia da educação: seis abordagens. São Paulo: Avercamp, p. 135-155, 2004.

MONTIEL-OVERALL, P. Cultural competence in web-based instruction: a conceptual framework. International Journal of Web Based Communities, 6(3), 254268, 2010.

PEÑA-AYALA, Alejandro (Ed.). Educational data mining: applications and trends. Springer, 2014. 
PORTER, C. E. A typology of virtual communities: A multi- disciplinary foundation for future research. Journal of Computer- Mediated Communication, 10(1), 2004.

ROGOFF, B. Apprenticeship in thinking: Cognitive development in sociocultural activity. New York: Oxford University Press, 1990.

ROGOFF, B. Developing understanding of the idea of communities of learners. Mind, culture, and activity, 1(4), 209-229, 1994.

ROGOFF, B. Observando a atividade sociocultural em três planos: apropriação participatória, participação guiada e aprendizado. EM WERTSCH, James V., Pablo DEL RIO, and Amelia ALVAREZ (Orgs), Estudos socioculturais da mente. Artmed: Porto Alegre, 123-142, 1998a.

ROGOFF, B. Cognition as a collaborative process. In W. Damon (Series Ed.), D. Kuhn, \& R. Siegler (Vol. Eds.), Handbook of child psychology: Vol. 2. Cognition, language, and perception (5th ed., pp. 679-744). New York: Wiley, $1998 \mathrm{~b}$.

ROGOFF, B. A natureza cultural do desenvolvimento humano. Porto Alegre: Artmed, 2005.

ROGOFF, B., MATUSOV, E., \& WHITE, C. Modelos de ensino e aprendizagem: a participação em uma comunidade de aprendizes. Educação e desenvolvimento humano. Porto Alegre: Artmed, 322-344, 2000.

SANTANA, S., ROAZZI, A., \& Maria das Graças, B. B.. Paradigmas do desenvolvimento cognitivo: uma breve retrospectiva. Estudos de psicologia, 11(1), 71-78, 2006.

SIEMENS, G., \& GASEVIC, D. Guest editorial-Learning and knowledge analytics. Educational Technology \& Society, 15(3), 1-2, 2012.

VIEIRA, C., PARSONS, P., BYRD, V. Visual learning analytics of educational data: A systematic literature review and research agenda. Computers \& Education, Elsevier, 2018.

VIRNOCHE, M. E., \& MARX, G. T. Only connect- E. M. Forster in an age of electronic communication: Computer- mediated association and community networks. Sociological Inquiry, 67 (1), 85-100, 1997.

WENGER, E. Communities of practice: Learning, meaning, and identity. New York: Cambridge University Press, 1998. 